05,12

\title{
Управляемый магнитными полями четырех наночастиц пиннинг доменной стенки в ферромагнитной нанопроволоке
}

\author{
() О.Л. Ермолаева ${ }^{1}$, Е.В. Скороходов ${ }^{1}$, В.Л. Миронов ${ }^{1,2}$ \\ ${ }^{1}$ Институт ффизики микроструктур РАН, \\ Нижний Новгород, Россия \\ ${ }^{2}$ Нижегородский государственный университет им. Н.И. Лобачевского, \\ Нижний Новгород, Россия \\ E-mail: ermolaeva@ipmras.ru
}

\begin{abstract}
Представлены результаты теоретических и экспериментальных исследований пиннинга доменной стенки в планарной ферромагнитной системе, представляющей собой нанопроволоку и четыре прямоугольные однородно намагниченные наночастицы, расположенные под углом к ней. На основании расчетов энергии взаимодействия доменной стенки с полями рассеяния наночастиц и микромагнитного моделирования продемонстрировано, что в такой системе реализуются различные варианты пиннинга доменной стенки, определяющиеся взаимной ориентацией магнитных моментов наночастиц и намагниченности нанопроволоки. Обсуждается возможность создания на основе рассмотренных структур магнитных логических ячеек.
\end{abstract}

Работа выполнена при поддержке РФФИ (гранты № 15-02-04462 и 15-42-02388р_поволжье_а).

\section{1. Введение}

Процессы пиннинга и депиннинга доменных стенок в ферромагнитных нанопроволоках являются предметом интенсивных исследований в связи с перспективностью использования таких систем для создания устройств магнитной памяти и магнитной логики [1-3]. Процесс вычислений в таких устройствах основан на управлении движением доменной стенки под действием внешнего магнитного поля или спин-поляризованного тока [4-6]. В связи с этим актуальными являются исследования, посвященные прецизионному позиционированию доменных стенок в нанопроволоках посредством создания локальных ловушек для контролируемого пиннинга/депиннинга. В частности, ряд исследований посвящен пиннингу доменных стенок на искусственных дефектах нанопроволок в виде выступов и впадин с различными формами [7-13]. Такие дефекты играют роль постоянных ловушек, а контроль движения доменных стенок в указанных системах осуществляется посредством изменения величины и длительности импульсов внешнего магнитного поля.

Альтернативный подход состоит в использовании для управления пиннингом доменной стенки магнитных полей рассеяния, создаваемых однородно намагниченными наночастицами, расположенными вблизи нанопроволоки [14-16]. Недавно нами были предложены два способа пиннинга доменной стенки в планарной ферромагнитной системе, состоящей из нанопроволоки и двух наночастиц, расположенных перпендикулярно [17] и параллельно [18] оси нанопроволоки. Было показано, что в этих случаях движение доменной стенки вдоль нанопроволоки связано с прохождением потенциального рельефа, состоящего из потенциальных ям и барьеров. При этом величина энергии пиннинга доменной стенки определяется взаимной ориентацией магнитных моментов в системе и может существенно меняться за счет изменения конфигурации магнитных моментов в подсистеме наночастиц. В настоящей работе приведены результаты исследований пиннинга доменной стенки в системе, представляющей собой нанопроволоку и четыре прямоугольные наночастицы, расположенные под углом к оси нанопроволоки. В частности, обсуждается возможность реализации на основе данной системы логической ячейки. Особенностью рассматриваемой логической ячейки является возможность изменения выполняемой логической операции.

\section{2. Эксперимент}

Исследуемая планарная система в виде нанопроволоки и четырех наночастиц была изготовлена методом электронной литографии и ионного травления. Многослойная структура $\mathrm{Ni}_{80} \mathrm{Fe}_{20}(20 \mathrm{~nm}) / \mathrm{V}(15 \mathrm{~nm}) / \mathrm{Cu}$ $(10 \mathrm{~nm})$ была нанесена на подложку Si при помощи магнетронного напыления. После этого структура покрывалась слоем фуллеренов $\mathrm{C}_{60}(80 \mathrm{~nm})$, который использовался в качестве электронного резиста. Начальная защитная маска формировалась в слое $\mathrm{C}_{60}$ при помощи системы ELPHY PLUS (на основе сканирующего электронного микроскопа „SUPRA 50VP“) с последующим химическим травлением в органическом растворителе. Затем изображение переносилось на слой $\mathrm{Cu}$ ионным травлением в $\mathrm{Ar}^{+}$и далее на слой $\mathrm{V}$ плазменным травлением в атмосфере фреона. На конечном этапе система, состоящая из нанопроволоки и наночастиц, была сформирована в слое $\mathrm{Ni}_{80} \mathrm{Fe}_{20}$ с помощью ионного травления в $\mathrm{Ar}^{+}$. Изображение структуры, полученное методом сканирующей электронной микроскопии (СЭМ), приведено на рис. 1.

Нанопроволока имела размер $3000 \times 100 \times 30 \mathrm{~nm}$, наночастицы имели размер $300 \times 100 \times 30 \mathrm{~nm}$, расстояние между частицами и нанопроволокой составляло $50 \mathrm{~nm}$. 


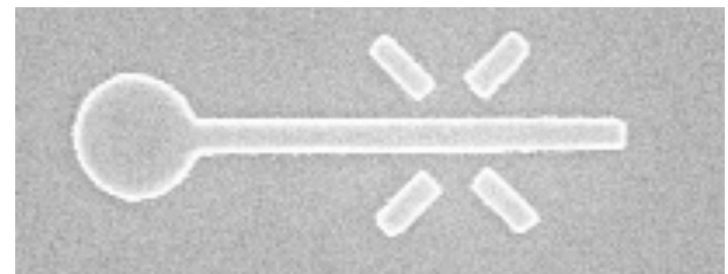

Рис. 1. СЭМ-изображение системы.

На одном конце нанопроволоки был сделан круглый диск диаметром $500 \mathrm{~nm}$, который обладал низкой коэрцитивностью и служил затравочной частью для зарождения доменной стенки. Частицы были расположены под углом $45^{\circ} \mathrm{C}$ к оси нанопроволоки. Процессы перемагничивания такой системы исследовались in situ с помощью магнитно-силового микроскопа (MCM) „Solver-HV“. Микроскоп оборудован электромагнитом, способным создавать поле в плоскости образца величиной до $1 \mathrm{kOe}$. Магнитно-силовые измерения проводились в вакууме $10^{-4}$ Torr. Использовался неконтактный режим сканирования. В качестве сигнала регистрировался сдвиг фазы колебаний кантилевера под действием градиента магнитного поля образца.

\section{3. Результаты}

Магнитные поля частиц создают сложный профиль потенциальной энергии для движения доменной стенки вдоль нанопроволоки, который существенно зависит от конфигурации магнитных моментов частиц. В рассматриваемой системе существует десять неэквивалентных магнитных состояний (рис. 2), обладающих различной величиной энергии депиннинга доменной стенки. Проведенный теоретический анализ потенциальной энергии системы и микромагнитное моделирование показали, что наибольшая энергия депиннинга соответствует конфигурациям магнитных моментов $S 1$ и $S 2$. Значение полей депиннинга в этом случае превышает характерное поле зарождения доменной стенки, составляющее по оценкам величину порядка 200 Ое. Конфигурации $S 3$ и $S 4$ также имеют достаточно высокий барьер депиннинга, однако эти конфигурации сложно реализовать на практике из-за высокой энергии магнитостатического взаимодействия между наночастицами [19]. Таким образом, конфигурации $S 1$ и $S 2$ перспективны для реализации магнитных логических ячеек. Остальные магнитные конфигурации имеют потенциальный барьер, характеризующийся более низкими значениями полей депиннинга.

На рис. 3 представлены зависимости энергии доменной стенки (типа „хвост к хвосту“) от ее положения в нанопроволоке. Видно, что в конфигурации $S 1$ (рис. 3, $a$ ) магнитное поле частиц создает потенциальный рельеф, состоящий из последовательно расположенных потенциальной ямы и барьера. Процесс движения доменной стенки в этом случае будет происходить следующим

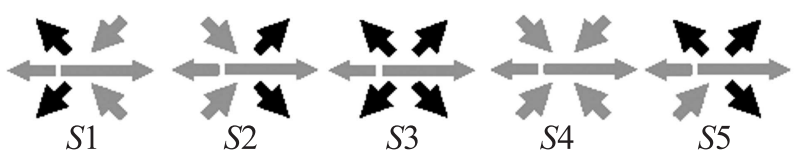

$\begin{array}{lllll}22.6 & 6.3 & 6.1 & 6.1 & 5.6\end{array}$

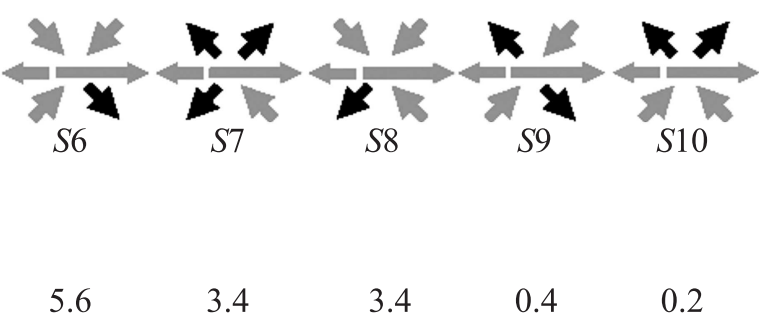

Рис. 2. Магнитные конфигурации системы и соответствующие величины энергии депиннинга (в единицах $10^{10} \mathrm{erg}$ ).
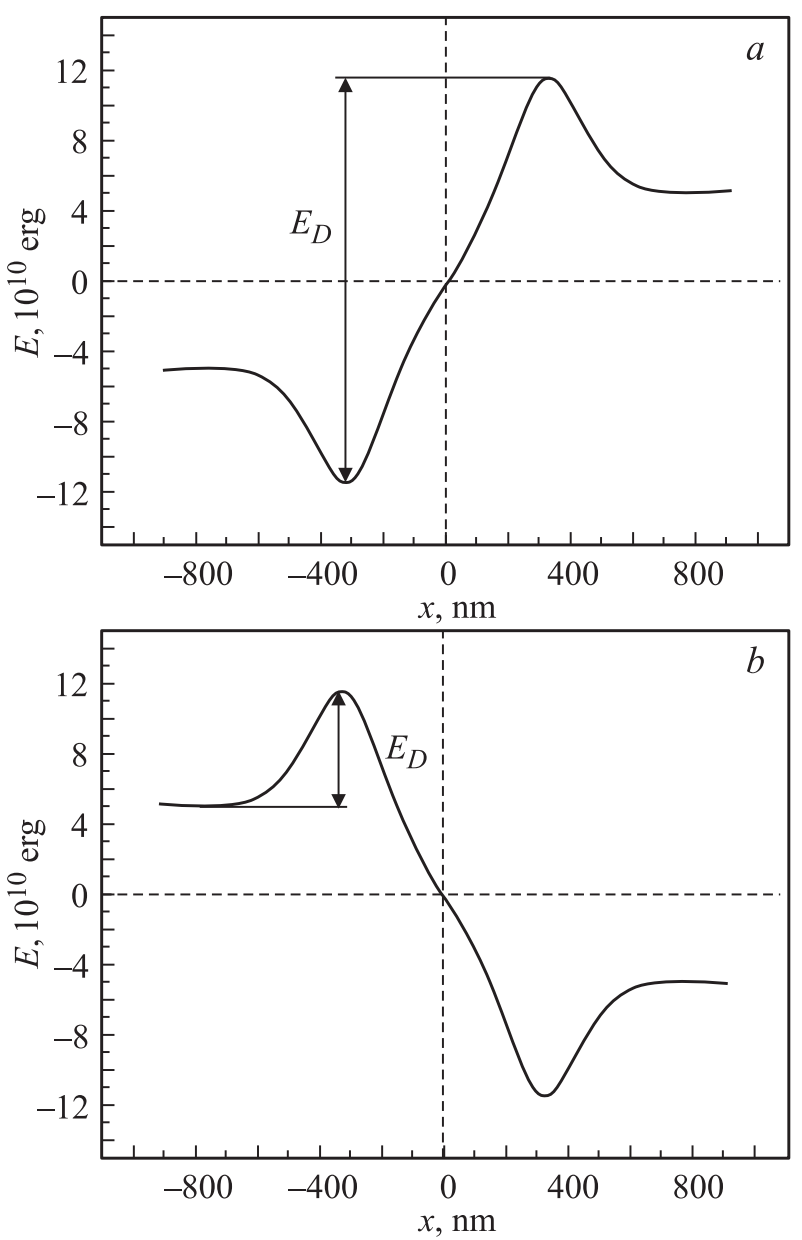

Рис. 3. Зависимости потенциальной энергии доменной стенки от ее положения в нанопроволоке. $a$ - потенциальный профиль для конфигурации $S 1 . b-$ потенциальный профиль для конфигурации $S 2$. Начало координат соответствует точке симметрии между частицами. 
образом. Доменная стенка возникает под действием внешнего магнитного поля в затравочной части и движется вдоль нанопроволоки (слева направо), пока не попадает в потенциальную яму, где она пиннингуется (энергия депиннинга $E_{D}$ ). Ее положение будет устойчиво до тех пор, пока внешнее магнитное поле не превысит значение поля депиннинга.

Другая ситуация наблюдается для конфигурации $S 2$. В этом случае потенциальный рельеф представляет собой последовательно расположенные и равные по абсолютной величине барьер и яму (рис. $3, b$ ). Как видно из рисунка, в этом случае величина энергии депиннинга равна высоте потенциального барьера и меньше, чем для магнитной конфигурации $S 1$. Кроме того, в отличие от предыдущего случая при конфигурации $S 2$ доменная стенка пиннингуется на потенциальном барьере, а не в яме, и в отсутствие внешнего магнитного поля она будет выталкиваться обратно из области наночастиц полями рассеяния.

Нами были проведены эксперименты по наблюдению процесса прохождения доменной стенки в описанной системе под действием внешнего магнитного поля. В каче-
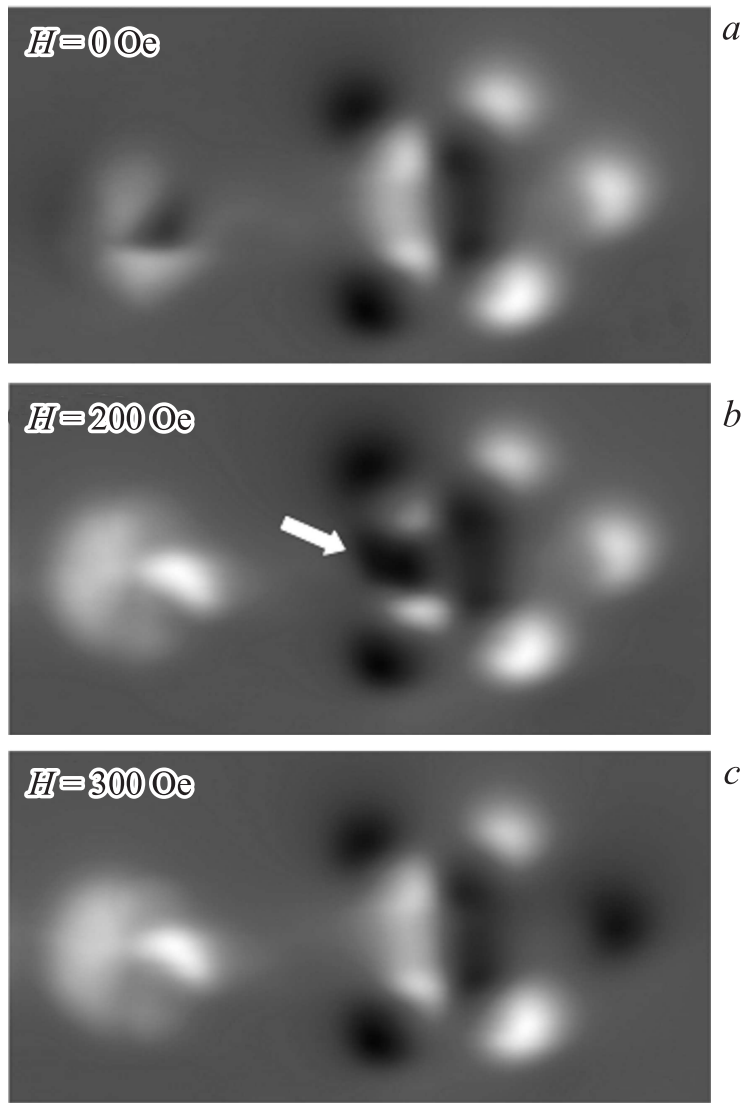

Рис. 4. МСМ-изображения последовательных стадий перемагничивания системы в состоянии $S 1$. $a-$ начальное состояние, нанопроволока намагничена однородно, $b-$ пиннинг доменной стенки (показана светлой стрелкой) в области между наночастицами после приложения поля 200 Ое. $c$ - депиннинг доменной стенки после приложения поля 300 Ое. Нанопроволока намагничена в противоположном направлении.

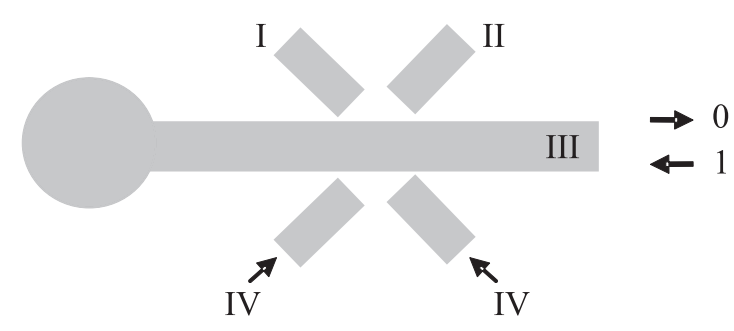

Pис. 5. Схема магнитной логической ячейки. I - вход 1, II - вход 2, III - выход, IV - управляющие частицы.

стве примера на рис. 4 представлены МСМ-изображения последовательных стадий перемагничивания системы для конфигурации магнитных моментов $S 1$.

Начальное состояние системы создавалось посредством намагничивания в сильном поле, приложенном вдоль оси нанопроволоки (рис. 4, a). Затем прикладывалось постепенно увеличивающееся поле, направленное в противоположную сторону. В поле 200 Ое в затравочной части происходило зарождение доменной стенки, которая под действием магнитного поля двигалась по нанопроволоке и пиннинговалась в области между первыми наночастицами. На МСМ-изображении (рис. 4, b) доменная стенка видна как дополнительный темный полюс. Положение доменной стенки было устойчивым вплоть до значения поля 300 Ое. При превышении этого поля наблюдалось перемагничивание нанопроволоки целиком, о чем можно судить по изменению цвета полюса на конце нанопроволоки (рис. $4, c$ ).

Соотношение величин полей зарождения доменной стенки и полей депиннинга в различных магнитных конфигурациях позволяет реализовать на основе данной системы магнитную логическую ячейку с переключаемой логической функцией (рис. 5). Основным элементом такой ячейки является ферромагнитная нанопроволока, по которой под действием внешнего магнитного поля движется доменная стенка (для определенности рассмотрим

Таблица 1. Таблица истинности логической операции „И“

\begin{tabular}{c|c|c}
\hline Вход 1 & Вход 2 & Выход \\
\hline 0 & 0 & 0 \\
0 & 1 & 0 \\
1 & 0 & 0 \\
1 & 1 & 1
\end{tabular}

Таблица 2. Таблица истинности логической операции „Или-Не“

\begin{tabular}{c|c|c}
\hline Вход 1 & Вход 2 & Выход \\
\hline 0 & 0 & 1 \\
0 & 1 & 0 \\
1 & 0 & 0 \\
1 & 1 & 0
\end{tabular}


доменную стенку типа „хвост к хвосту“). Пара частиц по одну сторону нанопроволоки - входные элементы ячейки. Свободный конец нанопроволоки является выходом ячейки. Вторая пара частиц служит для изменения логической операции, выполняемой ячейкой. Входная и выходная информация кодируется направлением магнитных моментов. Если магнитные моменты управляющих частиц направлены вправо, то такая логическая ячейка выполняет логическую операцию „И“ (табл. 1). В том случае, когда магнитные моменты управляющих частиц направлены влево, логическая ячейка выполняет логическую операцию „Или-Не“ (табл. 2). Если магнитные моменты управляющих частиц направлены в противоположные стороны, то такая ячейка выключена и не выполняет никакой логической операции.

Алгоритм работы такой ячейки аналогичен алгоритму, описанному в [19]. На первой стадии в нанопроволоке записывается логическая единица при помощи намагничивания ее во внешнем поле. После этого за счет локального магнитного поля записывается информация на входы и на управляющие наночастицы. На финальной стадии происходит процесс вычисления, заключающийся в приложении реверсивного магнитного поля с амплитудой меньше поля депиннинга и считывание выходной информации.

\section{4. Заключение}

Таким образом, в настоящей работе рассмотрены возможности пиннинга доменной стенки в системе, состоящей из нанопроволоки и четырех наночастиц, расположенных под углом $45^{\circ}$ к ее оси. Показано, что величина поля депиннинга в такой системе существенно зависит от взаимной ориентации магнитных моментов наночастиц и намагниченности нанопроволоки. Проанализированы магнитные конфигурации системы наночастиц, различающиеся величиной энергии пиннинга доменной стенки и соответственно значениями полей депиннинга. Показана принципиальная возможность реализации на основе такой системы перестраиваемой магнитной логической ячейки.

Авторы выражают благодарность С.Н. Вдовичеву и B.В. Рогову за помощь в изготовлении образцов, а также А.А. Фраерману за полезные обсуждения.

\section{Список литературы}

[1] D.A. Allwood, G. Xiong, C.C. Faulkner, D. Atkinson, D. Petit, R.P. Cowburn. Science 309, 1688 (2005).

[2] S.S.P. Parkin, M. Hayashi, L. Thomas. Science 320, 190 (2008).

[3] R.L. Stamps, S. Breitkreutz, J. Akerman, A.V. Chumak, Y. Otani, G.E.W. Bauer, J.-U. Thiele, M. Bowen, S.A. Majetich, M. Klaui, I.L. Prejbeanu, B. Dieny, N.M. Dempsey, B. Hillebrands. J. Phys. D: Appl. Phys., 47, 333001 (2014).

[4] D.A. Allwood, G. Xiong, M.D. Cooke, C.C. Faulkner, D. Atkinson, N. Vernier, R.P. Cowburn. Science 296, 2003 (2002).
[5] L. O'Brien, D.E. Read, H.T. Zeng, E.R. Lewis, D. Petit, R.P. Cowburn. Appl. Phys. Lett. 95, 232502 (2009).

[6] M. Hayashi, L. Thomas, C. Rettner, R. Moriya, Ya.B. Bazaliy, S.S.P. Parkin. Phys. Rev. Lett. 98, 037204 (2007).

[7] M. Hayashi, L. Thomas, R. Moriya, C. Rettner, S.S.P. Parkin. Science 320, 209 (2008).

[8] E. R. Lewis, D. Petit, L. Thevenard, A.V. Jausovec, L. O'Brien, D.E. Read, R.P. Cowburn. Appl. Phys. Lett. 95, 152505 (2009).

[9] D. Petit, A.V. Jausovec, D.E. Read, R.P. Cowburn. J. Appl. Phys. 103, 114307 (2008).

[10] D. Petit, A.V. Jausovec, H.T. Zeng, E.R. Lewis, L. O’Brien, D.E. Read, R.P. Cowburn. Phys. Rev. B 79, 214405 (2009).

[11] K. O'Shea, S. Mc Vitie, J.N. Chapman, J.M.R. Weaver. Appl. Phys. Lett. 93, 202505 (2008).

[12] L.K. Bogart, D. Atkinson, K. O'Shea, D. Mc Grouther, S. Mc Vitie. Phys. Rev. B 79, 054414 (2009).

[13] E. Varga, G. Csaba, A. Imre, W. Porod. IEEE Trans. Nanotechnol. 11, 682 (2012).

[14] Q. Zhu, X. Liu, S. Zhang, Q. Zheng, J. Wang, Q. Liu. Jpn. J. Appl. Phys. 53, 073001 (2014).

[15] S.M. Ahn, K.W. Moon, C.G. Cho, S.B. Choe. Nanotechnology 22, 085201 (2011).

[16] R.A. van Mourik, C.T. Rettner, B. Koopmans, S.S.P. Parkin. J. Appl. Phys. 115, 17D503 (2014).

[17] V.L. Mironov, O.L. Ermolaeva, E.V. Skorohodov, A.Yu. Klimov. Phys. Rev. B 85, 144418 (2012).

[18] В.Л. Миронов, О.Л. Ермолаева. Изв. РАН. Сер. физ. 78, 1, 36 (2015).

[19] A. Remhof, A. Schumann, A. Westphalen, H. Zabel. Phys. Rev. B 77, 134409 (2008). 